\title{
Feasible Test Case Generation Using Search Based Technique
}

\author{
Jasmine Minj \\ Dept. of Comp. Sc. and Engg., \\ Motilal National Institute of Technology, Allahabad
}

\begin{abstract}
This paper presents automatic test case generation technique. Multi population genetic algorithm is used to generate test cases. Fitness function is based on the multiple condition decision coverage criteria. MATLAB Gatool is used for implementing the test case generation algorithm. It generates efficient and effective test cases. Test cases are optimized using multi population genetic algorithm. MCDC coverage is used as coverage criteria. Automatic test cases generation reduce the testing effort, time and cost.
\end{abstract}

\section{General Terms:}

Test Case Generation

\section{Keywords:}

Multi population genetic algorithm, Multiple condition decision coverage

\section{INTRODUCTION}

Software testing is one of the important phase in software development. It is one of the critical task as it lead to increase in cost, time and effort. To make testing easy there is a need to generate test cases to guide the testing process. Generating an optimized test cases lead to improve the software efficiency. Search based technique is used for test case generation as testing is an exhausting process and it is very difficult to handle, and generating test cases is NP hard problem [11].

The proposed method for automatic test case generation following a criteria of MCDC coverage guided by multi population genetic algorithm. Coverage criteria is used to measure the extent to which a given system satisfy its goal. Multiple condition decision coverage criteria is used which should satisfy as follows:

(1) Every decision should execute all true as well as false criteria.

(2) Every condition should execute all true as well false criteria.

(3) Every condition in decision should be independently derive the output.

It is much more stricter than decision coverage. Using MCDC as coverage criteria we are able to evaluate software verification with a good metrics.

\section{RELATED WORK}

Hamilton Gross et al. [3] presented the evaluation of use of evolutionary testing technique in testing. Predicate coverage was used as coverage criteria. Java program taken as test data. Ali M. Alakeel [4] proposed the use of heuristic approach for test case generation based on assertion. Code based coverage criteria was used. Abdul Rauf et al. [1] presented an analysis of testing graphical user interface using genetic algorithm. Fitness function used as a evaluation parameter. Nodepad is taken as case study. Kewen Li et al. [2] proposed a method by combining genetic algorithm with particle swarm optimization to generate test cases efficiently. Diversity used as evaluation parameter and triangle program taken as case study.

Mark Harman et al. [5] presented three algorithm: Memory based, greedy based, CDG based approach to generate reduced and optimized test case which lead to reduced oracle cost. Yang Cao et al. [6] proposed genetic based automatic test case generation approach, fitness function on the basis of target path similarity with execution path. X. B. Tan et al. [7] proposed GA based annealing immune algorithm for generation of test data and presented the individual concentration adjustment and new mechanism for reproduction and triangle problem taken as case study. Yong Chen et al. [8] presented an evaluation of performance of branch distance based fitness function and modified hamming distance based fitness fucntion. And showed that branch based fitness function works more efficiently than modified hamming distance fitness function. For evaluation triangle problem taken as case study.

Praveen Ranjan Srivastava et al. [9] proposed a method to generate test cases effectively using ant colony algorithm and GA. Based on constraints and test data evaluated the performance of both technique in software testing. Susan Khor et al. [10] presented a automatic test case generation approach using GA with formal techniques. Statement coverage and data flow coverage used as a coverage criteria. A Fibonacci series based algorithm was proposed for selection process in test case generation.

\section{GENETIC ALGORITHM}

General procedure of genetic algorithm:

begin

INITIALIZE the population;

EVALUATE each individual;

repeat 
SELECT individuals;

APPLY CROSSOVER to the individual;

APPLY MUTATION to the resulting individual;

EVALUATE resulting individual;

SELECT individuals for the next generation

until STOPPING-CONDITION is reached

end

Initialization: Generated paths are represented in the form bit string and taken as initial chromosome for initial population.

Selection: Selection of individual is done on the basis of Fitness function based on Multiple condition decision coverage.

Crossover: It important part of GA. Crossover recombine bits of strings and produce the new result for next generation. We have used single point cross over. We have used crossover probability of 0.8 . We generate random number according to the present chromosome, random number less then 0.8 are selected for crossover.

Example:

Parent1: 526724

Parent2: 843531

After applying single point crossover:

Child 1:523531

Child 2: 846724

Mutation: Mutation gives the diversity to the population. We generate random number on the basis of current chromosome and mutation is applied if random number is less then the mutation probability of 0.2

\section{PROPOSED APPROACH}

Path based test case generation is used with search based technique for which it is converted into problem of optimization. It is the process of finding the best possible solution from the feasible solution. Multi population genetic algorithm is used as a search technique. It takes multiple populations. Randomly initialization of sub-population is done. Different Sub-population works separately with fitness function and have different search in search space. Then after some period of time they exchange their information and works again separately.

Method for test cases generation: Convert the program into Control flow graph. Generate all possible paths. Construct the fitness function based on MCDC coverage to evaluate the path feasibility. For example $\mathrm{X} \wedge \mathrm{y}$ where $\mathrm{X}, \mathrm{Y}$ are the condition in predicate. The MCDC test cases for this will be Case1: when $\mathrm{X}$ is True and $\mathrm{Y}$ is True then result should be True. Case2: when $X$ is True and $Y$ is False then result should be False. Case3: when $\mathrm{X}$ is False and $\mathrm{Y}$ is True then result should be False.. Construct the instrumental version of the program. And set the MATLAB Gatool tool to set the specified parameters for multi population genetic algorithm and perform the execution until the stopping criteria is reached.

\section{CASE STUDY}

A bench mark problem triangle problem is taken as case study. A Triangle program used is given three input sides of the triangle and output will be a type of triangle according to the given input value. A triangle function is shown to check the triangle type. It is con- verted into control flow graph shown in Fig. 1. Fitness function refer to the [12] branch distance function shown in Table 1 for predicates. The four path is shown here, out of which we find the most critical path and set the parameter in MATLAB Gatool for multi population genetic algorithm.

(1) Path: d

(2) Path: ae

(3) Path: abf

(4) Path: abc

Triangle Program:

function triangletype $=$ triangle $(\mathrm{X}, \mathrm{Y}, \mathrm{Z})$

if $(\mathrm{X}+\mathrm{Y}\rangle \mathrm{Z}) \&(\mathrm{Y}+\mathrm{Z}\rangle \mathrm{X}) \&(\mathrm{Z}+\mathrm{X}\rangle \mathrm{Y}) \&(\mathrm{X}\rangle 0) \&(\mathrm{Y}\rangle 0) \&(\mathrm{Z}\rangle 0)$

if $(\mathrm{X} \sim=\mathrm{Y}) \&(\mathrm{Y} \sim=\mathrm{Z}) \&(\mathrm{Z} \sim=\mathrm{X})$

triangletype='Scalene';

else

if $(\mathrm{X}==\mathrm{Y}) \&(\mathrm{Y} \sim=\mathrm{Z})\|(\mathrm{Y}==\mathrm{Z}) \&(\mathrm{Z} \sim=\mathrm{X})\|(\mathrm{Z}==\mathrm{X}) \&(\mathrm{X} \sim=\mathrm{Y})$

triangletype $=$ 'Isosceles';

else

triangletype='Equilateral';

end

end

else

type

triangletype='Not-a-triangle';

end

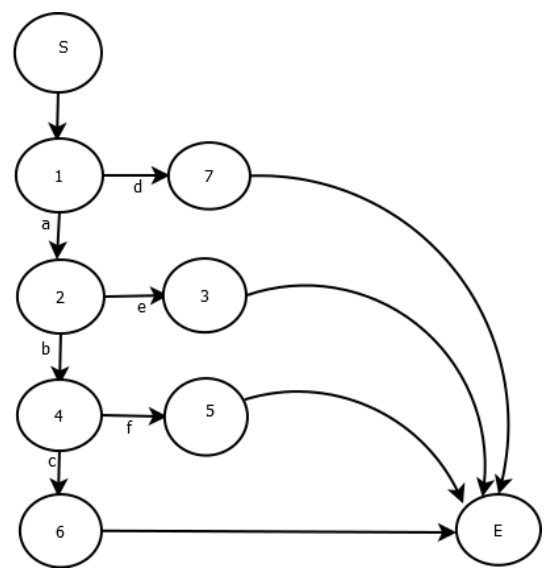

Fig. 1. Control flow graph of triangle problem

Matlab Gatool settings for MGPA:

(1) Binary string is taken as coding

(2) Population size $=2500$

(3) Number of sub-populations $=5$

(4) Number of individuals in each sub-population $=500$

(5) Fitness: Rank-based

(6) Selection:Roulette wheel 
Table 1.

Korel's_branch_distance

\begin{tabular}{|c|c|}
\hline Relational and logical Predicate: $\mathbf{C}$ & Distance if different path is taken \\
\hline $\mathrm{p} 1=\mathrm{p} 2$ & $\mathrm{abs}(\mathrm{p} 1-\mathrm{p} 2)$ \\
$\mathrm{p} 1 \neq \mathrm{p} 2$ & $\mathrm{~K}$ \\
$\mathrm{p} 1<\mathrm{p} 2$ & $(\mathrm{p} 1-\mathrm{p} 2)+\mathrm{k}$ \\
$\mathrm{p} 1 \leq \mathrm{p} 2$ & $\mathrm{p} 1-\mathrm{p} 2$ \\
$\mathrm{p} 1>\mathrm{p} 2$ & $(\mathrm{p} 2-\mathrm{p} 1)+\mathrm{k}$ \\
$\mathrm{p} 1 \geq \mathrm{p} 2$ & $\mathrm{p} 2-\mathrm{p} 1$ \\
$\mathrm{X} \wedge \mathrm{Y}$ & $\min (\operatorname{Distance}(\mathrm{X})+\operatorname{Distance}(\mathrm{Y}))$ \\
$\mathrm{X} \vee \mathrm{Y}$ & $(\operatorname{Distance}(\mathrm{X})+\operatorname{Distance}(\mathrm{Y}))$ \\
\hline
\end{tabular}

(7) Crossover: Single-point and probability $=0.8$

(8) Mutation probability $=0.2$

(9) Migration rate $=0.2$

(10) Maximum generation $=500$

(11) Insertion rate $=0.8$

(12) Generation gap $=0.8$

(13) Migration period $=20$

\section{RESULT AND ANALYSIS}

Experiment is done in MATLAB using Gatool by taking triangle problem as case study. Multi population genetic algorithm with multiple condition decision coverage shown a better performance that Multi population genetic algorithm with decision coverage shown in Table. 2. Performance evaluation of other example is also shown the benefits of proposed approach.

\section{CONCLUSION}

Path oriented test case generation is guided by multi-population genetic to generate test cases efficiently. Fitness function is made by following MCDC coverage. Triangle problem is taken as case study. Using MCDC approach with MPGA gives the good result in generating test cases efficiently. It generates feasible and optimized test cases.

\section{REFERENCES}

[1] Abdul Rauf, Sajid Anwar, M. Arfan Jaffer, Arshad Ali Shahid, "Automated GUI test coverage analysis using GA", 7-th International conference on Information Technology, 2010.

[2] Kewen Li, Zilu Zhang, Jisong Kou, "Breeding software test data with genetic-particle swarm mixed algorithm", Journal of Computers, Vol.5, No.2, Feb 2010.

[3] Hamilton Gross, Peter M. Kruse, Dr. Joachim Wegener, Dr. Tanja Vos, "Evolutionary white-box software test with the evotest framework, a progress report", Proceedings of the International Conference on Software Testing, Verification and Validation Workshop, 2010.

[4] Ali M. Alakeel, "An algorithm for efficient assertions-based test data generation", Proceedings of the International Multiconference of Engineers and Computer Scientists, Vol 5, No 6, pp. 644-653, June 2010.

[5] Mark Harman, Sung Gon Kim, Kiran Lakhotia, P. McMinn, Shin Yoo, "Optimizing for the number of tests generated in search based test data generation with an application to the oracle cost problem", Third International Conference on Software Testing, Verification, and Validation Workshops, 2010.

[6] Yang Cao, Chunhua Hu, Luming Li, "An Approach to generate software test data for a specific path automatically with genetic algorithm", 8th IEEE International Conference on Reliability, Maintainability and Safety, pp. 888-892, 2009.

[7] X. B. Tan, Cheng Longxin, Xu Xiumei, "Test data generation using annealing Immune genetic algorithm”, IEEE Computer Society, pp. 344-348,2009.

[8] Yong Chen1, Yong Zhong, Tingting Shi1, Jingyong Liu, "Comparison of two fitness functions for GA-based pathoriented test data generation ", Fifth International Conference on Natural Computation , 2009.

[9] Praveen Ranjan Srivastava, Vinod Ramachandran, Manish Kumar, Gourab Talukder, "Generation of test data using meta heuristic approach", Proceedings of IEEE Region Tenth Conference on TENCON, 2008.

[10] Susan Khor and Peter Grogono, "Using a Genetic algorithm and formal concept analysis to generate branch coverage test data automatically", IEEE Proceedings of the Nineteenth International Conference on Automated Software Engineering, 2009.

[11] Maha Alzabidi, Ajay Kumar, and A.D. Shaligram, "Automatic software structural testing by using evolutionary algorithms for test data generations", International Journal of Computer Science and Network Security, Vol.9, No.4, April 2009.

[12] B. Korel, "Automated software test data generation", IEEE Transactions of software Engineering, No.16, pp. 870-879, 1990 
Table 2.

Performance_Evaluation

\begin{tabular}{|c|c|c|c|c|c|}
\hline Program & Type & NO.of branches & Generation & Coverage percentage & Average time in second \\
\hline Triangle & MPGA with DC & 8 & 85 & 100 & 295.4 \\
Triangle & MPGA with MCDC & 8 & 25 & 100 & 25.3 \\
Quadratic & MPGA with DC & 6 & 95 & 100 & 395.6 \\
Quadratic & MPGA with MCDC & 6 & 35 & 100 & 35.2 \\
\hline
\end{tabular}

